\title{
Physical Wigner functions
}

\author{
Carlos L. Benavides-Riveros ${ }^{1,2,3}$ and José M. Gracia-Bondía ${ }^{2,3}$ \\ ${ }^{1}$ Zentrum für interdisziplinäre Forschung, Wellenberg 1 Bielefeld 33615, Germany \\ ${ }^{2}$ Departamento de Física Teórica, Universidad de Zaragoza, 50009 Zaragoza, Spain \\ ${ }^{3}$ Instituto de Biocomputación y Física de Sistemas Complejos (BIFI), Universidad de Zaragoza, 50018 Zaragoza, Spain
}

(Received 15 November 2012; published 20 February 2013)

\begin{abstract}
In spite of their potential usefulness, the characterizations of Wigner functions for Bose and Fermi statistics given by O'Connell and Wigner himself almost thirty years ago [Phys. Rev. A 30, 2613 (1984)] have drawn little attention. With an eye towards applications in quantum chemistry, we revisit and reformulate them in a more convenient way.
\end{abstract}

DOI: 10.1103/PhysRevA.87.022118

\section{INTRODUCTION AND HISTORY}

By definition, a spinless $n$-body Wigner quasiprobability distribution (or Wigner function for short) is given in terms of the density matrix in configuration or momentum space, respectively, $\rho, \widehat{\rho}$, by $[1]$

$$
\begin{aligned}
W_{\rho}(\boldsymbol{x} ; \boldsymbol{p}) & :=\frac{1}{\pi^{d n}} \int \rho(\boldsymbol{x}-\boldsymbol{z} ; \boldsymbol{x}+\boldsymbol{z}) e^{2 i \boldsymbol{p} \cdot \boldsymbol{z}} d \boldsymbol{z} \\
& =\frac{1}{\pi^{d n}} \int \widehat{\rho}(\boldsymbol{p}-\boldsymbol{z} ; \boldsymbol{p}+\boldsymbol{z}) e^{-2 i \boldsymbol{x} \cdot \boldsymbol{z}} d \boldsymbol{z},
\end{aligned}
$$

with the notation $\boldsymbol{x}=\left(\boldsymbol{x}_{1}, \ldots, \boldsymbol{x}_{n}\right)$ for $n$ bodies, where $\boldsymbol{x}_{i} \in \mathbb{R}^{d}$ (say $d=3$ for ordinary space), and similarly for $\boldsymbol{p}$ and $z$. For a pure state one has $\rho\left(\boldsymbol{x} ; \boldsymbol{x}^{\prime}\right)=\Psi(\boldsymbol{x}) \Psi^{*}\left(\boldsymbol{x}^{\prime}\right)$, with $\Psi$ the corresponding wave function. We have taken units so that $\hbar=1$. The relation $\rho \leftrightarrow W_{\rho}$ is one to one, being the restriction to the convex set of positive kernels of unit trace of a linear isomorphism of functions of two sets of variables, essentially the inverse of the unitary Wigner transformation. ${ }^{1}$

Averages of Wigner functions with classical phase-space observables reproduce the expected values predicted by standard quantum mechanics. This is why they have become an important tool, successfully adopted in statistical physics, quantum optics [3], and now chemistry [4,5]. It is however not easy to characterize them, although necessary and sufficient conditions for a phase-space function to be an admissible Wigner quasiprobability distribution are known [4,6,7].

Now, a natural question is: when does a Wigner function correspond to a wave function symmetric or antisymmetric under permutations of its variables? This was posed since the early days, in view of applications: see the references in Ref. [8]. But only the latter article purported to offer a general answer. $^{2}$

On their characterization, O'Connell and Wigner wrote: "It must be admitted that this equation for the distribution function, postulating the Bose statistics for a system of spin- 0 particles, is much more complicated that the corresponding equation for the density matrix." After discussing one-half spin systems, towards the conclusion of the paper, twice more they repeat this gloomy assessment almost verbatim.

\footnotetext{
${ }^{1}$ Várilly and one of us proved this transformation in Ref. [2] to be of order 24.

${ }^{2}$ We leave aside the second-quantized approach to Wigner quasiprobability, which has known scant success.
}

PACS number(s): 03.65.Sq, 71.10.-w, 05.30.Fk

It is true that their key formulas (14a) or (14b), together with their Eq. (11), look rather unwieldy. This moreover seems to have discouraged borrowing of the second and more interesting part of their paper, on systems of spin $1 / 2$ particles, hardly exploited elsewhere. They offered no examples.

Of late, development of density functional theory based on 1-body Wigner functions led us to reexamine the matter. ${ }^{3}$ We give here a simple answer to the question of quantum statistics for Wigner functions. We will be dealing mainly with identical fermions, for which the Wigner function is a spin multiplet; hence definition (1) will be insufficient. Even so, our characterizations take the form of mere preservations or changes of sign under permutation of two variables-just as in the ordinary formalism of quantum mechanics. This makes it plain that such permutations squared induce the identity, a fact not at all obvious in Ref. [8].

The summary of the article is as follows: We deal first with the conditions for symmetric or antisymmetric spinless Wigner functions-both are required for quantum chemistry purposes. We illustrate our contentions with a few example classes of concrete Wigner functions in Sec. III. Then we go on to Wigner spin orbitals, revisiting the second part of Ref. [8]. In Sec. V we exemplify again. Section VI is the conclusion.

\section{THE BASIC THEOREM}

It will be enough to consider the 2-body problem. Bringing in mean and difference coordinates, or, in chemists' jargon, extracule and intracule coordinates, respectively given by

$$
\boldsymbol{R}=\frac{1}{\sqrt{2}}\left(\boldsymbol{x}_{1}+\boldsymbol{x}_{2}\right), \quad \boldsymbol{r}=\frac{1}{\sqrt{2}}\left(\boldsymbol{x}_{1}-\boldsymbol{x}_{2}\right),
$$

with the customary abuse of notation, the symmetry or antisymmetry conditions (say, on configuration space) for spinless bodies respectively read $\rho\left(\boldsymbol{R}, \boldsymbol{r} ; \boldsymbol{R}^{\prime}, \boldsymbol{r}^{\prime}\right)= \pm \rho\left(\boldsymbol{R},-\boldsymbol{r} ; \boldsymbol{R}^{\prime}, \boldsymbol{r}^{\prime}\right)$ or $\rho\left(\boldsymbol{R}, \boldsymbol{r} ; \boldsymbol{R}^{\prime}, \boldsymbol{r}^{\prime}\right)= \pm \rho\left(\boldsymbol{R}, \boldsymbol{r} ; \boldsymbol{R}^{\prime},-\boldsymbol{r}^{\prime}\right)$. Together they imply

$$
\rho\left(\boldsymbol{R}, \boldsymbol{r} ; \boldsymbol{R}^{\prime}, \boldsymbol{r}^{\prime}\right)=\rho\left(\boldsymbol{R},-\boldsymbol{r} ; \boldsymbol{R}^{\prime},-\boldsymbol{r}^{\prime}\right)
$$

\footnotetext{
${ }^{3}$ Their $\mathrm{N}$-representability conditions, including nonensemble aspects $[9,10]$, are well understood. Existence and some of the properties of the energy functional with Wigner functions as variables were established in Ref. [4]. The theory has the flavour of an almost exact Thomas-Fermi formalism in phase space, needing "only" to incorporate electron correlation.
} 
and, reciprocally, the latter indistinguishability property together with either of the above conditions implies the other.

It is not hard to see that with

$$
\boldsymbol{P}=\frac{1}{\sqrt{2}}\left(\boldsymbol{p}_{1}+\boldsymbol{p}_{2}\right), \quad \boldsymbol{p}=\frac{1}{\sqrt{2}}\left(\boldsymbol{p}_{1}-\boldsymbol{p}_{2}\right),
$$

the meaning of $W(\boldsymbol{R}, \boldsymbol{r} ; \boldsymbol{P}, \boldsymbol{p})$ is unambiguous. This is due to the linear symplectic invariance of the Wigner function formalism. Then (3) is equivalent to

$$
W(\boldsymbol{R}, \boldsymbol{r} ; \boldsymbol{P}, \boldsymbol{p})=W(\boldsymbol{R},-\boldsymbol{r} ; \boldsymbol{P},-\boldsymbol{p}) .
$$

Since the discussion turns around the intracule variables, it is worth regarding $\boldsymbol{R}, \boldsymbol{P}$ as parameters, introducing the following notation:

$$
\omega_{\boldsymbol{R}, \boldsymbol{P}}(\boldsymbol{r}, \boldsymbol{p}):=W(\boldsymbol{R}, \boldsymbol{r} ; \boldsymbol{P}, \boldsymbol{p}) .
$$

Let us now invoke the following partial Fourier transform on the intracule set of variables:

$$
\tilde{\omega}_{\boldsymbol{R}, \boldsymbol{P}}(\boldsymbol{v}, \boldsymbol{p}):=\int \omega_{\boldsymbol{R}, \boldsymbol{P}}(\boldsymbol{r}, \boldsymbol{p}) e^{2 i \boldsymbol{v} \cdot \boldsymbol{r}} d \boldsymbol{r}=\tilde{\omega}_{\boldsymbol{R}, \boldsymbol{P}}(-\boldsymbol{v},-\boldsymbol{p}) .
$$

The last equality is seen to hold when (3) or equivalently (5) hold, and reciprocally. Presently, we have two momentum-like intracular variables, and the following appears natural:

Theorem 1. A spinless Wigner 2-body function comes from a density matrix symmetric or antisymmetric in its first set of variables (respectively, in its second set) if and only if, for all $\boldsymbol{v}$ and $\boldsymbol{p}$,

$$
\begin{aligned}
\tilde{\omega}_{\boldsymbol{R}, \boldsymbol{P}}(\boldsymbol{v}, \boldsymbol{p}) & = \pm \tilde{\omega}_{\boldsymbol{R}, \boldsymbol{P}}(\boldsymbol{p}, \boldsymbol{v}) \\
{\left[\text { respectively, } \tilde{\omega}_{\boldsymbol{R}, \boldsymbol{P}}(\boldsymbol{v}, \boldsymbol{p})\right.} & \left.= \pm \tilde{\omega}_{\boldsymbol{R}, \boldsymbol{P}}(-\boldsymbol{p},-\boldsymbol{v})\right] .
\end{aligned}
$$

Proof. Consider the following integral:

$$
\begin{aligned}
\tilde{\omega}_{\boldsymbol{R}, \boldsymbol{P}}(\boldsymbol{v}, \boldsymbol{p})= & \frac{1}{\pi^{2 d}} \int \rho(\boldsymbol{R}-\boldsymbol{Z}, \boldsymbol{r}-\boldsymbol{z} ; \boldsymbol{R}+\boldsymbol{Z}, \boldsymbol{r}+\boldsymbol{z}) \\
& \times e^{2 i \boldsymbol{P} \cdot \boldsymbol{Z}+2 i \boldsymbol{p} \cdot \boldsymbol{z}} e^{2 i \boldsymbol{v} \cdot \boldsymbol{r}} d \boldsymbol{Z} d z d \boldsymbol{r} \\
= & \frac{ \pm 1}{\pi^{2 d}} \int \rho(\boldsymbol{R}-\boldsymbol{Z}, \boldsymbol{z}-\boldsymbol{r} ; \boldsymbol{R}+\boldsymbol{Z}, \boldsymbol{z}+\boldsymbol{r}) \\
& \times e^{2 i \boldsymbol{P} \cdot \boldsymbol{Z}+2 i \boldsymbol{v} \cdot \boldsymbol{r}} e^{2 i \boldsymbol{p} \cdot \boldsymbol{z}} d \boldsymbol{Z} d \boldsymbol{r} d \boldsymbol{z} \\
= & \pm \int \omega_{\boldsymbol{R}, \boldsymbol{P}}(\boldsymbol{z}, \boldsymbol{v}) e^{2 i \boldsymbol{p} \cdot \boldsymbol{z}} d \boldsymbol{z}=: \pm \tilde{\omega}_{\boldsymbol{R}, \boldsymbol{P}}(\boldsymbol{p}, \boldsymbol{v}) .
\end{aligned}
$$

Thus necessity of the first condition is proved. Conversely, given that $\rho \leftrightarrow W_{\rho}$ is one-to-one, it is readily seen that (6) holds only if $\rho$ is respectively symmetric or antisymmetric. The proof of the second condition is similar. Clearly, if we assume $\tilde{\omega}_{\boldsymbol{R}, \boldsymbol{P}}(\boldsymbol{v}, \boldsymbol{p})=\tilde{\omega}_{\boldsymbol{R}, \boldsymbol{P}}(-\boldsymbol{v},-\boldsymbol{p})$, either of the conditions of (6) implies the other.

Our result extends to $n$-body functions by just considering intracule and extracule coordinates for the first pair of adjacently labeled particles. That is, we require only one condition of the kind (6), together with the indistinguishability condition (5) for all intracules.

\section{EXAMPLES}

Use of Gaussian basis sets in density functional theory with Wigner functions is if anything more natural than in standard quantum chemistry [11]. This motivates our first example.
Example 1. Take as a boson-type wave function the symmetric product of two general Gaussians centered at the origin:

$$
\Psi\left(x_{1}, x_{2}\right)=C\left[\psi_{1}\left(x_{1}\right) \psi_{2}\left(x_{2}\right)+\psi_{1}\left(x_{2}\right) \psi_{2}\left(x_{1}\right)\right],
$$

where, for $j=1,2$,

$$
\psi_{j}(x)=\frac{d_{j}^{1 / 4}}{\pi^{1 / 4}} e^{-\frac{1}{2} d_{j} x^{2}-\frac{i}{2} b_{j} d_{j} x^{2}} \quad \text { with } \quad d_{j}>0, b_{j} \in \mathbb{R} .
$$

(The normalization factor $C$ is unimportant here.) The corresponding 2-body quasidensity is

$$
\begin{aligned}
W\left(x_{1}, x_{2} ; p_{1}, p_{2}\right) \propto & W_{11}\left(x_{1} ; p_{1}\right) W_{22}\left(x_{2} ; p_{2}\right) \\
& +W_{22}\left(x_{1} ; p_{1}\right) W_{11}\left(x_{2} ; p_{2}\right) \\
& +W_{12}\left(x_{1} ; p_{1}\right) W_{21}\left(x_{2} ; p_{2}\right) \\
& +W_{21}\left(x_{1} ; p_{1}\right) W_{12}\left(x_{2} ; p_{2}\right) .
\end{aligned}
$$

Here $W_{j k}$ represents an interference; namely,

$$
W_{j k}(x, p)=\frac{d_{j}^{1 / 4} d_{k}^{1 / 4}}{\pi d_{j k}^{1 / 2}} e^{-A_{j k} x^{2}-2 B_{j k} x p-d_{j k}^{-1} p^{2}},
$$

where

$$
\begin{aligned}
d_{j k} & :=\frac{1}{2}\left(d_{j}+d_{k}\right)+\frac{i}{2}\left(b_{j} d_{j}-b_{k} d_{k}\right), \\
b_{j k} & :=\frac{1}{2}\left(b_{j} d_{j}+b_{k} d_{k}\right)-\frac{i}{2}\left(d_{j}-d_{k}\right), \\
A_{j k} & :=d_{j k}+b_{j k}^{2} / d_{j k}, \\
B_{j k} & :=b_{j k} / d_{j k} .
\end{aligned}
$$

The quadratic form in the exponent of the $W_{j k}$ is given by a symmetric, symplectic matrix with positive definite real part [4]. When $k=j$, we have a Gaussian pure state,

$$
W_{j j}(x, p)=\pi^{-1} e^{-\left(d_{j}+b_{j}^{2} d_{j}\right) x^{2}-2 b_{j} x p-d_{j}^{-1} p^{2}},
$$

whose coefficient matrix is real, symplectic, and positive definite [12].

To see that the quasidensity (8) fulfils (6), change variables according to Eqs. (2) and (4) and let $\lambda_{i j k l}(R, r ; P, p):=$ $W_{i j}\left(x_{1} ; p_{1}\right) W_{k l}\left(x_{2} ; p_{2}\right)$. Now, multiplying by $e^{2 i v r}$ and integrating with respect to $r$, we obtain, after a little work,

$$
\begin{aligned}
\int \lambda_{j j k k}(R, r ; P, p) e^{2 i v r} d r & =\int \lambda_{j k k j}(R, r ; P, v) e^{2 i p r} d r \\
\text { with } \quad k & \neq j,
\end{aligned}
$$

thereby verifying condition (6) for this example. Mutatis mutandis, Gaussian sets like the ones in (7) with a minus instead of a plus sign exemplify the antisymmetric case.

Example 2. In the early years of quantum mechanics, as a prolegomenon to calculating the energy levels for helium, Heisenberg [13] studied the harmonium, an exactly integrable analog of a two-electron atom. It exhibits two fermions interacting with an external harmonic potential and repelling each other by a Hooke-type force. Being simple, but not trivial, this system has been borrowed in many contexts. It is sometimes called the "Moshinsky atom," since Moshinsky reintroduced it with the purpose of studying correlation energy $[5,14]$. Also, it has been recruited to investigate black-hole entropy [15], Bose-Einstein condensation [16], fundamental issues in interference experiments [17], and sundry ones in quantum chemistry-see Refs. [10,18,19] and references therein. 

by

The harmonium Hamiltonian in Hartree-like units is given

$$
\begin{aligned}
& H\left(\boldsymbol{x}_{1}, \boldsymbol{x}_{2} ; \boldsymbol{p}_{1}, \boldsymbol{p}_{2}\right) \\
& \quad=\frac{\left|\boldsymbol{p}_{1}\right|^{2}}{2}+\frac{\left|\boldsymbol{p}_{2}\right|^{2}}{2}+\frac{k}{2}\left(\left|\boldsymbol{x}_{1}\right|^{2}+\left|\boldsymbol{x}_{2}\right|^{2}\right)-\frac{\delta}{4}\left|\boldsymbol{x}_{1}-\boldsymbol{x}_{2}\right|^{2} .
\end{aligned}
$$

Introducing extracule and intracule coordinates and the frequencies $v:=\sqrt{k}$ and $\mu:=\sqrt{k-\delta}$, the Hamiltonian is rewritten as that of two independent oscillators:

$$
H=H_{R}+H_{r}:=\frac{|\boldsymbol{P}|^{2}}{2}+\frac{\nu^{2}|\boldsymbol{R}|^{2}}{2}+\frac{|\boldsymbol{p}|^{2}}{2}+\frac{\mu^{2}|\boldsymbol{r}|^{2}}{2} .
$$

Since the problem factorizes completely, we work in dimension one. The orbital part of such an eigenfunction is written $\phi_{n}(R) \psi_{m}(r)$, with the parity of $\psi_{m}(r)$ even for spin singlet states and odd for triplet states. Wigner quasiprobabilities associated with those eigenvectors have the general form $W_{n}(R, P) W_{m}(r, p)$, where, with $L_{n}$ denoting the $n$th Laguerre polynomial,

$$
\begin{aligned}
W_{n}(R, P) & =\frac{(-1)^{n}}{\pi} L_{n}\left(4 H_{R} / v\right) e^{-2 H_{R} / v}, \\
W_{m}(r, p) & =\frac{(-1)^{m}}{\pi} L_{m}\left(4 H_{r} / \mu\right) e^{-2 H_{r} / \mu} .
\end{aligned}
$$

Defining

$$
\begin{aligned}
\Gamma_{m}(v, p) & =(-1)^{m} \int W_{m}(r, p) e^{2 i v r} d r \\
& =\frac{1}{\pi} \int L_{m}\left(4 H_{r} / \mu\right) e^{-2 H_{r} / \mu} e^{2 i v r} d r,
\end{aligned}
$$

by use of the generating function of the Laguerre polynomials, we obtain

$$
\begin{aligned}
& \sum_{m=0}^{\infty} \Gamma_{m}(v, p) x^{m} \\
& \quad=\frac{1}{\pi(1-x)} \int e^{-4\left(H_{r} / \mu\right) x /(1-x)} e^{-2\left(H_{r} / \mu\right)} e^{2 i v r} d r
\end{aligned}
$$

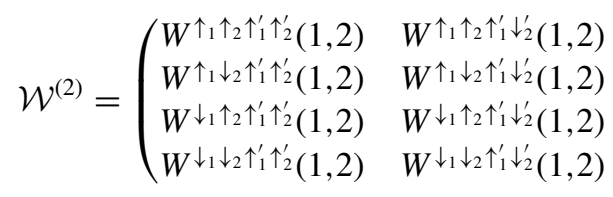$$
W^{\uparrow_{1} \uparrow_{2} \downarrow_{1}^{\prime} \uparrow_{2}^{\prime}}(1,2)
$$$$
W^{\uparrow_{1} \downarrow_{2} \downarrow_{1}^{\prime} \uparrow_{2}^{\prime}}(1,2)
$$$$
W \downarrow_{1} \uparrow_{2} \downarrow_{1}^{\prime} \uparrow_{2}^{\prime}(1,2)
$$$$
W^{\downarrow_{1} \downarrow_{2} \downarrow_{1}^{\prime} \uparrow_{2}^{\prime}}(1,2)
$$

$$
\begin{aligned}
& =\frac{1}{\pi(1-x)} e^{-\frac{1+x}{1-x} p^{2} / \mu} \int e^{-\frac{1+x}{1-x} \mu r^{2}+2 i v r} d r \\
& =\frac{1}{\sqrt{\pi \mu\left(1-x^{2}\right)}} \exp \left(-\frac{1+x}{1-x} \frac{p^{2}}{\mu}-\frac{1-x}{1+x} \frac{v^{2}}{\mu}\right) \\
& =\sum_{m=0}^{\infty}(-)^{m} \Gamma_{m}(p, v) x^{m} .
\end{aligned}
$$

Thus $\Gamma_{m}(v, p)=-\Gamma_{m}(p, v)$ for $m$ odd and $\Gamma_{m}(v, p)=$ $\Gamma_{m}(p, v)$ for $m$ even, and whenever the wave function $\Psi_{n m} \equiv \phi_{n} \psi_{m}$ is symmetric or antisymmetric, the corresponding Wigner functions $W_{n m}(R, P ; r, p)=W_{n}(R, P) W_{m}(r, p)$ in agreement with (6) do respectively satisfy

$$
\int W_{n m}(R, P ; r, p) e^{2 i v r} d r= \pm \int W_{n m}(R, P ; r, v) e^{2 i p r} d r .
$$

\section{SPIN WIGNER FUNCTIONS}

The standard definition for spin- $\frac{1}{2}$ Wigner functions, found for instance in the seminal work on atomic Wigner functions [20], regards the latter (just as the density matrices) as $2^{n} \times 2^{n}$ matrices in spin space:

$$
\begin{aligned}
& \mathcal{W}_{\rho}^{\varsigma_{1}, \ldots, \varsigma_{n} ; \varsigma_{1}^{\prime}, \ldots, \varsigma_{n}^{\prime}}\left(\boldsymbol{x}_{1}, \ldots, \boldsymbol{x}_{n} ; \boldsymbol{p}_{1}, \ldots, \boldsymbol{p}_{n}\right) \\
& \quad:=\frac{1}{\pi^{d n}} \int \rho\left(\boldsymbol{x}-\boldsymbol{z} ; \varsigma_{1}, \ldots, \varsigma_{n} ; \boldsymbol{x}+\boldsymbol{z}, \varsigma_{1}^{\prime}, \ldots, \varsigma_{n}^{\prime}\right) e^{2 i \boldsymbol{p} \cdot \boldsymbol{z}} d \boldsymbol{z} .
\end{aligned}
$$

Here $\varsigma$ and $\varsigma^{\prime}$ denote the discrete spin variables. In particular, a 1-body atomic Wigner distribution in matrix form would be of the form

$$
\mathcal{W}^{(1)}=\left(\begin{array}{ll}
W^{\uparrow_{1} \uparrow_{1^{\prime}}}(\boldsymbol{x} ; \boldsymbol{p}) & W^{\uparrow_{1} \downarrow_{1^{\prime}}(\boldsymbol{x}, \boldsymbol{p})} \\
W^{\downarrow_{1} \uparrow_{1^{\prime}}(\boldsymbol{x}, \boldsymbol{p})} & W^{\downarrow_{1} \downarrow_{1^{\prime}}(\boldsymbol{x}, \boldsymbol{p})}
\end{array}\right),
$$

and a 2-body atomic Wigner distribution is

redundancies in practice. It was implicitly criticized by Wigner in his last years [8,21]. He sought instead to endow the spin Wigner functions with ostensible physical meaning, by arranging their entries into tensors under the rotation group.

Given the essentially unitary matrix,

$$
U:=\frac{1}{2}\left(\sigma_{\varsigma \varsigma^{\prime}}^{\kappa}\right)=\frac{1}{2}\left(\begin{array}{cccc}
1 & 0 & 0 & 1 \\
0 & 1 & 1 & 0 \\
0 & i & -i & 0 \\
1 & 0 & 0 & -1
\end{array}\right),
$$

where

$$
\kappa=0, x, y, z \quad \text { and } \quad U U^{\dagger}=1 / 2 \text {, }
$$

The matrix approach does not display the angular momentum structure of the reduced functions, and contains severe 
for the 1-body quasiprobability those are provided by

$$
\left(\begin{array}{l}
W^{0} \\
W^{x} \\
W^{y} \\
W^{z}
\end{array}\right)=U\left(\begin{array}{l}
W^{\uparrow_{1} \uparrow_{1^{\prime}}} \\
W^{\uparrow_{1} \downarrow_{1^{\prime}}} \\
W^{\downarrow_{1} \uparrow_{1^{\prime}}} \\
W^{\downarrow_{1} \downarrow_{1^{\prime}}}
\end{array}\right)
$$

There the entries on the right-hand side are not real in general; but on the left side they are. Matters turn interesting for the 2-body function, whereupon

$$
\left(\begin{array}{l}
W^{0} \\
W^{x} \\
W^{y} \\
W^{z}
\end{array}\right) \otimes\left(\begin{array}{l}
W^{0} \\
W^{x} \\
W^{y} \\
W^{z}
\end{array}\right)=(U \otimes U)\left[\left(\begin{array}{l}
W \uparrow_{1} \uparrow_{1^{\prime}} \\
W^{\uparrow_{1} \downarrow_{1^{\prime}}} \\
W^{\downarrow_{1} \uparrow_{1^{\prime}}} \\
W^{\downarrow_{1} \downarrow_{1^{\prime}}}
\end{array}\right) \otimes\left(\begin{array}{l}
W^{\uparrow_{2} \uparrow_{2^{\prime}}} \\
W_{2}^{\uparrow_{2} \downarrow_{2^{\prime}}} \\
W^{\downarrow_{2} \uparrow_{2^{\prime}}} \\
W^{\downarrow_{2} \downarrow_{2^{\prime}}}
\end{array}\right)\right] .
$$

The central question addressed by O'Connell and Wigner is the transformation of $U \otimes U$ under particle exchange $1 \leftrightarrow 2$. This is better answered in terms of the physical tensor components of $\mathcal{W}$. Denoting representations of the rotation group by their dimension, and since the 1-body function is the sum of one rotational scalar and one vector part, the addition rule for angular momentum yields

$$
([\mathbf{1}] \oplus[\mathbf{3}])^{\otimes 2}=2[\mathbf{1}] \oplus 3[\mathbf{3}] \oplus[\mathbf{5}] ;
$$

that is, two scalars, three vectors, and one quadrupole (symmetric traceless tensor). Let now $W^{00}$ replace $W^{0} \otimes W^{0}$ in the notation, and so on. The particle exchange in the spin space $\varsigma_{1} \leftrightarrow \varsigma_{2}$ can be understood as a unitary transformation in the spin space; namely,

$$
\left.W^{\alpha \beta}\right|_{\varsigma_{1} \leftrightarrow \varsigma_{2}}:=\sum_{\gamma, \delta} B_{\gamma \delta}^{\alpha \beta} W^{\gamma \delta} \quad \text { with } \quad \gamma, \delta \in\{0, x, y, z\},
$$

and it is easy to show that

$$
B_{\gamma \delta}^{\alpha \beta}=\frac{1}{4} \operatorname{tr}\left[\left(\sigma^{\alpha} \sigma^{\gamma} \otimes \sigma^{\beta} \sigma^{\delta}\right) A\right] \quad \text { and } \quad B=B^{\dagger}=B^{-1} .
$$

Wigner and O'Connell suggest to take this matrix as the basic quantity of the theory. Its eigenvalues must be equal to \pm 1 . We diagonalize $B$ and observe that its eigenvectors, thus possessing (anti-) symmetric properties under particle exchange, are irreducible tensors under the rotation group. They constitute a basis for the space of 2-body Wigner distributions in spin space. In fine, we reorganize the left-hand side of Eq. (10) as the following spin multiplet:

$$
\begin{aligned}
W^{s c 1}= & W^{00}-W^{x x}-W^{y y}-W^{z z}, \\
W^{s c 2}= & \frac{1}{3}\left(3 W^{00}+W^{x x}+W^{y y}+W^{z z}\right), \\
W^{v 1}= & \left(W^{x 0}+W^{0 x}, W^{y 0}+W^{0 y}, W^{z 0}+W^{0 z}\right), \\
W^{v 2}= & \left(W_{-}^{x 0}+i W_{-}^{z y}, W_{-}^{y 0}+i W_{-}^{x z}, W_{-}^{0 z}+i W_{-}^{x y}\right), \\
W^{v 3}= & \left(W_{-}^{x 0}-i W_{-}^{z y}, W_{-}^{y 0}-i W_{-}^{x z}, W_{-}^{z 0}-i W_{-}^{y x}\right), \\
W^{q}= & \left(-W^{x x}-W^{y y}+2 W^{z z}, W^{x y}+W^{y x}, W^{y z}+W^{z y},\right. \\
& \left.W^{x x}-W^{y y}, W^{x z}+W^{z x}\right),
\end{aligned}
$$

with $W_{-}^{x 0}:=W^{x 0}-W^{0 x}, W_{-}^{x y}:=W^{x y}-W^{y x}$, and so on. The first two terms of the multiplet are the scalars, one symmetric and the other antisymmetric under spin particle exchange, then the three vectors, two symmetric and one antisymmetric, and the quadrupole (symmetric) in a standard presentation.
In summary, collecting $(s c 1, s c 2, v 1, v 2, v 3, q) \equiv \boldsymbol{f}$, for us an electronic 2-body Wigner function is the multiplet denoted $\omega(\boldsymbol{r}, \boldsymbol{p} ; \boldsymbol{f})$, the extracule labels being suppressed. The Fermi symmetry condition for the exchange of one set of spin coordinates $\varsigma_{1} \leftrightarrow \varsigma_{2}$ and of the spatial coordinates, borrowing the notation used in the spinless case, reads

$$
\tilde{\omega}(\boldsymbol{v}, \boldsymbol{p} ; \boldsymbol{f}):=-\tilde{\omega}\left(\boldsymbol{p}, \boldsymbol{v} ; \boldsymbol{f}_{\varsigma_{1} \leftrightarrow \varsigma_{2}}\right) .
$$

Then the exchange transformation rule for the Wigner function multiplet comes out, if anything, simpler, in that there are fewer minus signs than the one for the density matrix:

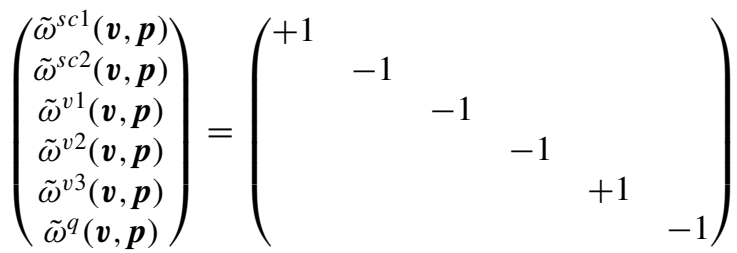

$$
\begin{aligned}
& \times\left(\begin{array}{c}
\tilde{\omega}^{s c 1}(\boldsymbol{p}, \boldsymbol{v}) \\
\tilde{\omega}^{s c 2}(\boldsymbol{p}, \boldsymbol{v}) \\
\tilde{\omega}^{v 1}(\boldsymbol{p}, \boldsymbol{v}) \\
\tilde{\omega}^{v 2}(\boldsymbol{p}, \boldsymbol{v}) \\
\tilde{\omega}^{v 3}(\boldsymbol{p}, \boldsymbol{v}) \\
\tilde{\omega}^{q}(\boldsymbol{p}, \boldsymbol{v})
\end{array}\right)
\end{aligned}
$$

This is because $\tilde{\omega}^{s c 1}$ is odd under $\varsigma_{1} \leftrightarrow \varsigma_{2}$, while $\tilde{\omega}^{s c 2}$ is even, and so on. Of course, one can choose to impose the Fermi condition on the primed spin coordinates. Then $\tilde{\omega}^{v 2}, \tilde{\omega}^{v 3}$ are peculiar in that they become respectively odd and even. ${ }^{4}$ But the general indistinguishability condition (9) now implies

$$
\begin{aligned}
& \left(\begin{array}{c}
\tilde{\omega}^{s c 1}(\boldsymbol{v}, \boldsymbol{p}) \\
\tilde{\omega}^{s c 2}(\boldsymbol{v}, \boldsymbol{p}) \\
\tilde{\omega}^{v 1}(\boldsymbol{v}, \boldsymbol{p}) \\
\tilde{\omega}^{v 2}(\boldsymbol{v}, \boldsymbol{p}) \\
\tilde{\omega}^{v 3}(\boldsymbol{v}, \boldsymbol{p}) \\
\tilde{\omega}^{q}(\boldsymbol{v}, \boldsymbol{p})
\end{array}\right)=\left(\begin{array}{cccccc}
+1 & & & & \\
& +1 & & & & \\
& & +1 & & & \\
& & & -1 & & \\
& & & & -1 & \\
& & & & & +1
\end{array}\right) \\
& \times\left(\begin{array}{c}
\tilde{\omega}^{s c 1}(-\boldsymbol{v},-\boldsymbol{p}) \\
\tilde{\omega}^{s c 2}(-\boldsymbol{v},-\boldsymbol{p}) \\
\tilde{\omega}^{v 1}(-\boldsymbol{v},-\boldsymbol{p}) \\
\tilde{\omega}^{v 2}(-\boldsymbol{v},-\boldsymbol{p}) \\
\tilde{\omega}^{v 3}(-\boldsymbol{v},-\boldsymbol{p}) \\
\tilde{\omega}^{q}(-\boldsymbol{v},-\boldsymbol{p})
\end{array}\right),
\end{aligned}
$$

and this saves the day.

\section{MORE EXAMPLES}

Formula (11) is well adapted to the needs of quantum chemistry since the standard Hamiltonian there does not contain spin coordinates; thus one uses a spin-restricted formalism [23], with the same set of symmetric or antisymmetric spatial orbitals for "up" and "down" spins. Then several components of the Wigner multiplet simply vanish. For a two-fermion

\footnotetext{
${ }^{4}$ The same behavior was observed by studying the spin structure for the 2-body reduced density matrix in Sec. 6A of Ref. [22].
} 
system, the singlet pure spin state is of the form

$$
\begin{aligned}
\mathcal{W}^{\text {singlet }}= & \frac{1}{2}\left(\uparrow_{1} \uparrow_{1^{\prime}} \downarrow_{2} \downarrow_{2^{\prime}}-\uparrow_{1} \downarrow_{1^{\prime}} \downarrow_{2} \uparrow_{2^{\prime}}-\downarrow_{1} \uparrow_{1^{\prime}} \uparrow_{2} \downarrow_{2^{\prime}}\right. \\
& \left.+\downarrow_{1} \downarrow_{1^{\prime}} \uparrow_{2} \uparrow_{2^{\prime}}\right) W=W^{s c 1} .
\end{aligned}
$$

The only nonzero contribution is given by the first scalar, and in the occasion the Pauli principle naturally reads $\tilde{\omega}^{\text {singlet }}(\boldsymbol{v}, \boldsymbol{p})=$ $\tilde{\omega}^{\text {singlet }}(\boldsymbol{p}, \boldsymbol{v})$. The one-body Wigner distribution for this state is just $W^{0}$. For triplet states, one deals with a linear superposition of symmetric spin states; namely,

$$
|\Psi\rangle=\alpha\left|\uparrow_{1} \uparrow_{2}\right\rangle+\beta \frac{\left|\uparrow_{1} \downarrow_{2}\right\rangle+\left|\downarrow_{1} \uparrow_{2}\right\rangle}{\sqrt{2}}+\gamma\left|\downarrow_{1} \downarrow_{2}\right\rangle,
$$

provided that

$$
|\alpha|^{2}+|\beta|^{2}+|\gamma|^{2}=1 .
$$

In terms of the Wigner spin multiplet, it reads

$$
\begin{aligned}
\mathcal{W}^{\text {triplet }}= & \left(|\alpha|^{2}+|\gamma|^{2}\right)\left(W^{s c 2}+\frac{1}{3} W^{q, 1}\right) \\
& +|\beta|^{2}\left(W^{s c 2}-\frac{2}{3} W^{q, 1}\right) \\
& +\frac{1}{\sqrt{2}}\left(\alpha^{*} \beta+\alpha \beta^{*}+\gamma^{*} \beta+\gamma \beta^{*}\right) W^{v 1, x} \\
& +\frac{i}{\sqrt{2}}\left(\alpha^{*} \beta-\alpha \beta^{*}-\gamma^{*} \beta+\gamma \beta^{*}\right) W^{v 1, y} \\
& +\left(|\alpha|^{2}-|\gamma|^{2}\right) W^{v 1, z}+\frac{i}{\sqrt{2}}\left(\alpha^{*} \beta-\alpha \beta^{*}\right. \\
& \left.+\gamma^{*} \beta-\gamma \beta^{*}\right) W^{q, 3}+i\left(\gamma \alpha^{*}-\gamma^{*} \alpha\right) W^{q, 2} \\
& +\left(\gamma \alpha^{*}+\gamma^{*} \alpha\right) W^{q, 4} \\
& +\frac{1}{\sqrt{2}}\left(\alpha^{*} \beta+\alpha \beta^{*}-\gamma^{*} \beta-\gamma \beta^{*}\right) W^{q, 5} .
\end{aligned}
$$

In the multiplet expansion of the triplet there appear only the second scalar, the first vector, and the quadrupole. They all carry minus signs in Eq. (11), and the transformation rule reads $\tilde{\omega}^{\text {triplet }}(\boldsymbol{v} ; \boldsymbol{p})=-\tilde{\omega}^{\text {triplet }}(\boldsymbol{p} ; \boldsymbol{v})$. Its one-body distribution is equal to

$$
\begin{aligned}
W^{0} & +\frac{1}{\sqrt{2}}\left(\alpha^{*} \beta+\alpha \beta^{*}+\gamma^{*} \beta+\gamma \beta^{*}\right) W^{x} \\
& +\frac{i}{\sqrt{2}}\left(\alpha^{*} \beta-\alpha \beta^{*}-\gamma^{*} \beta+\gamma \beta^{*}\right) W^{y}+\left(|\alpha|^{2}-|\gamma|^{2}\right) W^{z} .
\end{aligned}
$$

Notice how different are the $\beta=\gamma=0$ or $\beta=\alpha=0$ basic states, leading respectively to survival of $W^{00}+W^{z z} \pm$ $\left(W^{z 0}+W^{0 z}\right)$, from the $\alpha=\gamma=0$ state, to which there corresponds $W^{00}+W^{x x}+W^{y y}-W^{z z}$. That these states belong in different strata under rotations is somewhat hidden in the Hilbert space formalism-see the discussion in Sec. 7.7.c of Ref. [24]-but here it is apparent.

\section{CONCLUSION}

We hope to have thrown long-due light on the achievements of Ref. [8], helping to rescue its insights from near oblivion. On the theoretical side, the little attention received by that paper has concerned mostly its adaptation to the spherical phase-space Moyal formalism for spin, developed by Várilly and one of us in Ref. [25]. For a recent example, see Ref. [26]. As well, for a nice treatment of that formalism, emphasizing its connection to the tensor operators, see Ref. [27]. The middle path followed here, in the footsteps of Wigner and O'Connell, displays as well the physical appeal and information of the spherical method, while appearing better adapted to the needs of quantum chemistry. We can only speculate that it essentially coincides with the (so far, unpublished) approach "by the theory of group representations" arrived at by Moyal in his last years ([28], Ch. 8).

Needless to say, one may formulate all our results analogously in terms of distributions of the sort $\hat{\omega}_{\boldsymbol{R}, \boldsymbol{P}}(\boldsymbol{r}, \boldsymbol{s}):=$ $\int \omega_{\boldsymbol{R}, \boldsymbol{P}}(\boldsymbol{r}, \boldsymbol{p}) e^{-2 i \boldsymbol{s} \cdot \boldsymbol{p}} d \boldsymbol{p}$.

\section{ACKNOWLEDGMENTS}

We are grateful to Joseph C. Várilly for a careful reading of the manuscript. C. L. B. R. has been supported by a Banco Santander scholarship. J. M. G. B. has been supported by a grant from the regional government of Aragón. He is indebted as well to the Zentrum für interdisziplinäre Forschung for support and customarily warm hospitality.
[1] E. P. Wigner, Phys. Rev. 40, 749 (1932).

[2] J. C. Várilly and J. M. Gracia-Bondía, J. Math. Phys. 28, 2390 (1987).

[3] U. Leonhardt, Essential Quantum Optics (Cambridge University Press, Cambridge, 2010).

[4] Ph. Blanchard, J. M. Gracia-Bondía and J. C. Várilly, Int. J. Quantum Chem. 112, 1134 (2012).

[5] K. Ebrahimi-Fard and J. M. Gracia-Bondía, J. Math. Chem. 50, 440 (2012).

[6] F. J. Narcowich and R. F. O'Connell, Phys. Rev. A 34, 1 (1986).

[7] J. M. Gracia-Bondía and J. C. Várilly, Phys. Lett. A 128, 20 (1988).

[8] R. F. O'Connell and E. P. Wigner, Phys. Rev. A 30, 2613 (1984).

[9] A. A. Klyachko, J. Phys.: Conf. Ser. 36, 72 (2006).

[10] C. Schilling, D. Gross, and M. Christandl, Phys. Rev. Lett. 110, 040404 (2013).
[11] F. Jensen, Introduction to Computational Chemistry (Wiley, Chichester, 2007).

[12] R. G. Littlejohn, Phys. Rep. 138, 193 (1986).

[13] W. Heisenberg, Z. Phys. 38, 411 (1926).

[14] M. Moshinsky, Am. J. Phys. 36, 52 (1968).

[15] M. Srednicki, Phys. Rev. Lett. 71, 666 (1993).

[16] R. Seiringer, Ph.D. thesis, Universität Wien, 2000.

[17] W. M. Itano, J. C. Bergquist, J. J. Bollinger, D. J. Wineland, U. Eichmann, and M. G. Raizen, Phys. Rev. A 57, 4176 (1998).

[18] C. L. Benavides-Riveros, J. M. Gracia-Bondía, and J. C. Várilly, Phys. Rev. A 86, 022525 (2012).

[19] C. L. Benavides-Riveros and J. C. Várilly, Eur. Phys. J. D 66, 274 (2012).

[20] M. Springborg and J. P. Dahl, Phys. Rev. A 36, 1050 (1987).

[21] E. P. Wigner, in The Physics of Phase Space, edited by Y. S. Kim and W. W. Zachary (Springer, Berlin, 1987), pp. 162-170. 
[22] E. R. Davidson, Reduced Density Matrices in Quantum Chemistry (Academic Press, New York, 1976).

[23] M. Piris, Int. J. Quantum Chem. 113, 620 (2013).

[24] L. C. Biedenharn and J. D. Louck, Angular Momentum in Quantum Physics. Theory and Applications (Addison-Wesley, Reading, 1981).
[25] J. C. Várilly and J. M. Gracia-Bondía, Ann. Phys. (NY) 190, 107 (1989).

[26] P. Watson and A. J. Bracken, Phys. Rev. A 83, 042102 (2011).

[27] F. Li, C. Braun, and A. Garg, arXiv:1210.4075.

[28] A. Moyal, Maverick Mathematician: The Life and Science of J. E. Moyal (ANU E-press, Canberra, 2006). 